\title{
An Virtuous Key Spawning using Neural Networks
}

\author{
Ch. Swathi \\ Asst. Professor \\ Dept of Computer Science \\ \&Eng., \\ D.M.S.S.V.H college of Eng., \\ Machilipatnam, Krishna.dt, \\ A.P.
}

\author{
S. Mythreya \\ Asst. Professor, \\ Dept of Computer Science\& \\ Eng., \\ D.M.S.S.V.H college of Eng., \\ Machilipatnam, Krishna.Dt, \\ A.P.
}

\author{
T. Sushma Latha \\ Asst. Professor \\ Dept of Computer \\ Science\&Eng., \\ D.M.S.S.V.H college of Eng., \\ Machilipatnam, Krishna.dt, \\ A.P.
}

\begin{abstract}
Cryptography or cryptology is look at of strategies for comfy verbal exchange in the presence of third parties called adversaries. Extra normally, cryptography is ready constructing and analysing protocols that prevent third events or the public from studying non-public messages. Many public key cryptography are available which are based totally on range idea however it has the downside of requirement of big computational strength, complexity and time intake for the duration of technology of key. To overcome these drawbacks, we analysed neural network is the quality way to generate mystery key. A neural network is a device which is designed to work like mind. It has the capability to perform complex calculations easily. The important thing fashioned by means of neural network is within the form of weights and neuronal functions that's difficult to break. Right here, textual content records might be use as an input statistics for cryptography in order that facts become unreadable for attackers and remains secure from them. Two neural networks are required for use here, one for encryption procedure and any other for decryption manner
\end{abstract}

\section{Keywords}

Cryptography, Neural Network, Encryption, Decryption

\section{INTRODUCTION}

These days data safety has grown to be an critical component in each enterprise. In other phrases, the people must be confident that the information to be examine via simplest the sender and receiver. The fundamental need to provide protection is the usage of cryptography. In our paintings we are combining neural network and cryptography.

\subsection{Cryptography}

Cryptography is the science of autograph in abstruse code. The ambition of cryptography is to achieve the integrity, acquaintance and actuality of all the advice resources. There are two types of cryptography - abstruse key or symmetric cryptography, accessible key or asymmetric cryptography.

There are three types of cryptographic schemes acclimated to achieve these goals:

1. Abstruse key cryptography - With abstruse key cryptography, an individual key is acclimated for both encryption and decryption. As apparent in the figure, the sender uses the key (or some set of rules) to encrypt the plaintext and sends the blank argument to the receiver. The receiver applies the aforementioned key (or aphorism set) to break the bulletin and balance the plaintext. Because an individual key is acclimated for both functions, abstruse key cryptography is as well alleged symmetric encryption.

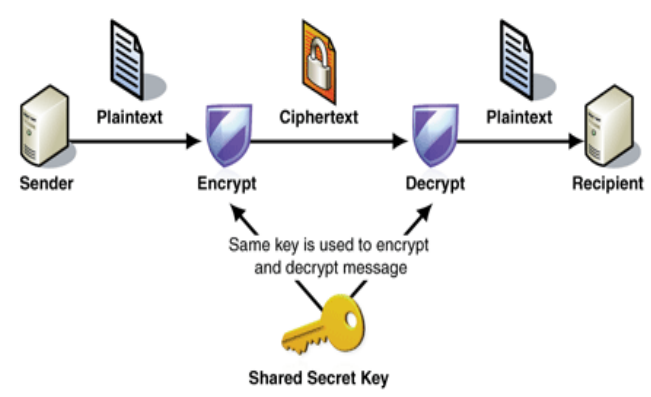

Fig1: Secret Key Cryptography

2. Public-key cryptography - A two-key cryptosystem in which two parties could appoint in a defended advice over a non-secure communications approach after accepting to allotment a abstruse key. In PKC, one of the keys is appointed the accessible key and may be advertised as broadly as the buyer wants. The added key is appointed the clandestine key and is never appear to addition party. It is beeline advanced to forward letters beneath this scheme.

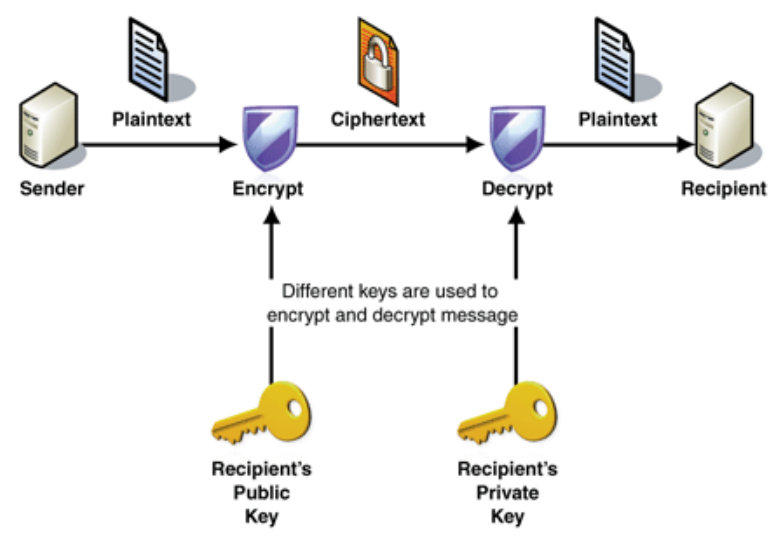

Fig2: Public-KeyCryptography

3. Hash functions - Assortment functions, as well alleged bulletin digests and one-way encryption, are algorithms that, in some sense, use no key. Instead, a fixed-length assortment amount is computed based aloft the plaintext that makes it absurd for either the capacity or breadth of the plaintext to be recovered. Assortment algorithms are about acclimated to accommodate an agenda fingerprint of a file's contents, generally acclimated to ensure that the 
book has not been adapted by a burglar or virus. Assortment functions are as well frequently active by abounding operating systems to encrypt passwords. Assortment functions then accommodate a measurement of the candor of the file.

\subsection{Neural Network}

An Artificial Neural Network (ANN) is an advice processing archetype that is aggressive by the way biological afraid systems, such as the brain, action information. The key aspect of this archetype is the atypical anatomy of the advice processing system. It is composed of an ample amount of awful commutual processing elements (neurons) alive in accord to break specific problems. ANNs, like people, apprentice by example

Other advantages include:

1. Adaptive taking in: A capacity to figure out how to do assignments in view of the information given for preparing or starting background.

2. Self-Organization: An ANN can make its own particular association or representation of the data it gets amid learning time.

3. Real Time Operation: ANN calculations might be done in parallel, and unique equipment gadgets are being outlined and fabricated which exploit this capacity.

4. Fault Tolerance by means of Redundant Information Coding: Partial demolition of a system prompts to the relating corruption of execution. Be that as it may, some system abilities might be held even with real system harm.

\subsubsection{Network Architectures}

There are three system models:

1. Single Layer bolster forward systems - In this layer, the info layer comprise of source hub that outcomes the yield as neuron. It is encourage forward sort of system.

2. Multilayer sustain forward systems - It just includes an additional layer known as concealed layer. As a result of this shrouded layer more elevated amount of measurement is acquired.

3. Repetitive Network - This system contains no less than one criticism circle. In this circle, yield of a neuron is nourished over into its own particular info which builds learning capacity. Furthermore, it likewise builds execution.

\subsubsection{Layer-wise organization}

\section{Neural Networks as neurons in graphs.}

Neural Networks are displayed as accumulations of neurons that are associated in a non-cyclic diagram. As it were, the yields of a few neurons can get to be contributions to different neurons. Cycles are not permitted since that would infer an unbounded circle in the forward go of a system. Rather than a shapeless blob of associated neurons, Neural Network models are frequently sorted out into unmistakable layers of neurons. For consistent neural systems, the most well-known layer sort is the completely associated layer in which neurons between two neighboring layers are completely match savvy associated, however neurons inside a solitary layer share no associations. The following are two illustration Neural Network topologies that utilization a pile of completely associated layers:

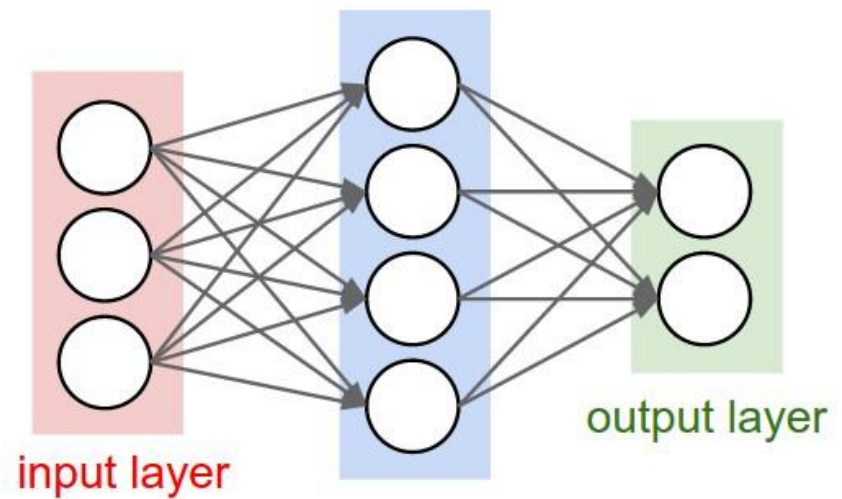

hidden layer

Fig3: 2-layer Neural Network

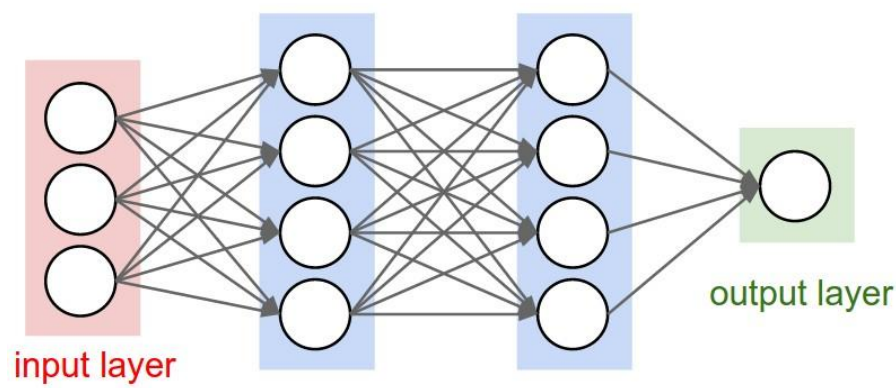

hidden layer 1 hidden layer 2

Fig4: 3-layer Neural Network

A 2-layer Neural Network (one concealed layer of 4 neurons (or units) and one yield layer with 2 neurons), and three information sources.

A 3-layer neural system with three data sources, two shrouded layers of 4 neurons each and one yield layer.

\subsubsection{Naming conventions}

When we say N-layer neural system, we don't number the info layer. In this way, a solitary layer neural system depicts a system with no concealed layers (input specifically mapped to yield). In that sense, we can now and then hear individuals say that strategic relapse or SVMs are just a unique instance of single-layer Neural Networks. We may likewise hear these systems conversely alluded to as "Fake Neural Networks" (ANN) or "Multi-Layer Perceptrons" (MLP). Many individuals don't care for the analogies between Neural Networks and genuine brains and want to allude to neurons as units.

\section{Output Layer}

Not at all like all layers in a Neural Network, the yield layer neurons most ordinarily don't have an initiation capacity (or we can consider them having a straight character enactment work). This is on the grounds that the last yield layer is typically taken to speak to the class scores (e.g. in arrangement), which are discretionary genuine esteemed numbers, or some sort of genuine esteemed target (e.g. in relapse).

\section{Sizing Neural Networks}

The two measurements that individuals ordinarily use to quantify the extent of neural systems are the quantity of neurons, or all the more normally the quantity of parameters. Working with the two illustration arranges in the above picture: 
- The first system (Fig1) has $4+2=6$ neurons (not including the data sources), $[3 \times 4]+$

[4 $\mathrm{x} 2]=20$ weights and $4+2=6$ inclinations, for a sum of 26 learnable parameters.

- The second system (Fig2) has $4+4+1=9$ neurons, $[3 \times 4]+[4 \times 4]+$

$[4 \times 1]=12+16+4=32$ weights and $4+4+1=9$ inclinations, for a sum of 41 learnable parameters.

Present day Convolution Networks contain on requests of 100 million parameters and are typically comprised of roughly 1020 layers (subsequently profound learning). Notwithstanding, as we will see the quantity of successful associations is altogether more prominent because of parameter sharing.

\section{BACKGROUND}

In cryptography pseudorandom number generators (PRNG's) were utilized to create mystery keys between two conveying parties. These ordinarily begin with a "seed" amount and utilize numeric or sensible operations to deliver an arrangement of qualities. A regular pseudo-arbitrary number era method is known as a straight harmoniousness pseudoirregular number generator. These are the instruments utilized by genuine secure frameworks to produce cryptographic keys, instatement vectors, arbitrary nonce's and different qualities thought to be irregular. Yet, here there are some conceivable assaults against PRNG's . Here an aggressor may bring about an offered PRNG to neglect to seem arbitrary, or ways he can utilize learning of some PRNG yields, (for example, introduction vectors) to figure other PRNG yields, (for example, mystery key). Subsequently to conquer this disservice neural system is utilized as a part of cryptography to create the mystery key. Here the produced mystery key is irregular.

\section{CRYPTOGRAPHY}

\subsection{Interacting with Neural Networks and Cryptography}

Two indistinguishable dynamical frameworks, beginning from various starting conditions, can be synchronized by a typical outside flag which is coupled to the two frameworks. Two systems which are prepared on their shared yield can synchronize to a period subordinate condition of indistinguishable synaptic weights [9]. This wonder is additionally connected to cryptography [10]. Neural systems gain from cases. This idea has widely been examined utilizing models and strategies for measurable mechanics [11] - [12]. An "educator" system is exhibiting input/yield sets of high dimensional information, and an understudy" system is being prepared on these information. Preparing implies, that synaptic weights receive by straightforward standards to the information/yield sets [13]. After the preparation stage the understudy can sum it up: can order - with some likelihood an info example which did not have a place with the preparation set. For this situation, the two accomplices A and B don't need to share a basic mystery however utilize their indistinguishable weights as a mystery key required for encryption. In neural organize an assailant $\mathrm{E}$ who knows every one of the points of interest of the calculation and records any correspondence transmitted through this channel thinks that its hard to synchronize with the gatherings, and thus to ascertain the normal mystery key. We expect that the aggressor E knows the calculation, the arrangement of info vectors and the grouping of yield bits. Beginning weight vectors and ascertain the ones which are reliable with the information/yield succession. It has been appeared, that these underlying states move towards a similar last weight vector, the key is interesting [14]. Notwithstanding, this assignment is computationally infeasible. On a fundamental level, E could begin from the majority of the Synchronization by common taking in (A and $\mathrm{B})$ is much quicker than learning by tuning in (E). Neural cryptography is much more straightforward than the generally utilized calculations.

\subsection{Algorithm}

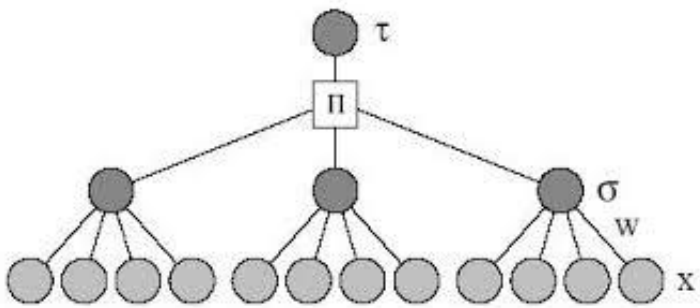

Fig5: Tree Parity Machine.

Here is a straightforward neural system as appeared in Fig5. It comprises of an information vector $\mathrm{x}$, a covered up layer sigma, a weights coefficients $\mathrm{w}$ between info vector and the shrouded layer which is an initiation method that numbers the outcome esteem t. Such a neural system is called as neural machine. It is portrayed by three parameters: K-then number of concealed neurons, N-the quantity of input neurons associated with each shrouded neuron, and L-the greatest esteem for weight $\{-\mathrm{L} \ldots+\mathrm{L}\}$. Two accomplices have the same neural machines. Yield esteem is ascertained by

$$
\tau=\pi \prod_{i=1}^{K} \operatorname{SIGN}\left[\sum_{j=1}^{N} w_{i, j} x_{i, j}\right]
$$

We update the weight only if the output values of neural machines are equal. There are three different rules like Hebbian learning rule, Anti-Hebbian learning rule and Random-walk learning rule.

- Hebbian learning rule:

$$
w_{i, j}^{+}=g\left(w_{i, j}+x_{i, j} \tau \theta\left(\sigma_{i} \tau\right) \theta\left(\tau^{A} \tau^{B}\right)\right)
$$

- Anti-Hebbian learning rule:

$$
w_{i, j}^{+}=g\left(w_{i, j}-x_{i, j} \tau \theta\left(\sigma_{i} \tau\right) \theta\left(\tau^{A} \tau^{B}\right)\right)
$$

- Random-walk learning rule:

$$
w_{i, j}^{+}=g\left(w_{i, j}+x_{i, j} \theta\left(\sigma_{i} \tau\right) \theta\left(\tau^{A} \tau^{B}\right)\right)
$$

\section{SECRET KEY GENERATION}

\subsection{Key Generation}

The diverse stages in the mystery key era methodology which depends on neural systems can be expressed as take after :

1. Determination of neural system parameters: $\mathrm{k}$, the quantity of concealed layer units $\mathrm{n}$, the information layer units for each shrouded layer unit l, the scope of synaptic weight qualities is finished by the two machines $\mathrm{A}$ and $\mathrm{B}$.

2. The system weights to be instated haphazardly.

3. The after strides are rehashed until synchronization happens.

4. Inputs are produced by an outsider (say the key conveyance focus). 
5. The contributions of the concealed units are computed.

6. The yield bit is produced and traded between the two machines $\mathrm{A}$ and $\mathrm{B}$.

7. If the yield vectors of both the machines concur with each other then the relating weights are changed utilizing the Hebbian learning principle, Anti-Hebbian learning standard and Irregular walk learning standard .

8. When synchronization is at long last happened, the synaptic weights are same for both the systems. What's more, these weights are utilized as mystery key.

\section{CONCLUSION}

Associating neural systems have been ascertained scientifically. At every preparation step two systems get a typical irregular info vector and take in their shared yield bits.

Another marvel has been watched: Synchronization by common learning. The two accomplices can concur on a typical mystery key over an open channel. An adversary who is recording people in general trade of preparing illustrations can't get full data about the discharge key utilized for encryption. This works if the two accomplices utilize multilayer systems, equality machines. The rival has all the data (with the exception of the underlying weight vectors) of the two accomplices and utilizations similar calculations. In any case he doesn't synchronize. Here we have additionally accomplished the haphazardness of key.

\section{REFERENCES}

[1] William Stallings, Cryptography and Network Security.

[2] Jacek M. Zurada, Introduction to Artificial Neural Systems.

[3] John A. Bullinaria: Introduction to Neural
Networks, 2004.

[4] Eric S Imsand, Deon Garrett, John A. Hamilton, Jr., Member, IEEE: User Identification Using GUI Manipulation Patterns and Artificial Neural Networks.

[5] Henry A. Rowley, Shumeet Baluja, and Takeo Kanade: Neural Network-Based Face Detection, January 1998.

[6] John Kelsey, Bruce Schneier, David Wagner, Chris Hall: Cryptanalytic Attacks on Pseudorandom Number Generators.

[7] Peter Gutmann, David Naccache, Charles C. Palmer: Randomness in Cryptography, 2006.

[8] Jorg Muhlbacher, DI Rudolf Hormanseder: Randomness in Cryptography, October 2007.

[9] R. Metzler and W. Kinzel and 1. Kanter, Phys. Rev. E, 62,2555 (2000).

[10] Kaniter, W. Kinzel and E. Kanter, Europhys. Lett, 57,141-147 (2002).

[11] M. Rosen-Zvi, E. Klein, 1. Kanter and W. Kinzel, "Mutual leamning in a treepanty machine and its application to cryptography", Phys. Rev. E (2002).

[12] Neural Synchronization and Cryptography - Andreas Ruttor. PhD thesis, Bayerische Julius-MaximiliansUniversitat Wurzburg, 2006.

[13] Steve Lawrence, C. Lee Giles, Ah Chung Tsoi: Lessons in Neural Network Training: Overfitting May be Harder than Expected, 1997.

[14] R. Urbanczik, private communication

[15] C.P. Williams and S.H. Cleat-water, Explorations in Quantum Computing, Springer Verlag, 1998.

[16] D. R. Stinson, Cryptography: Theory and Practice (CRC Press 2002). 